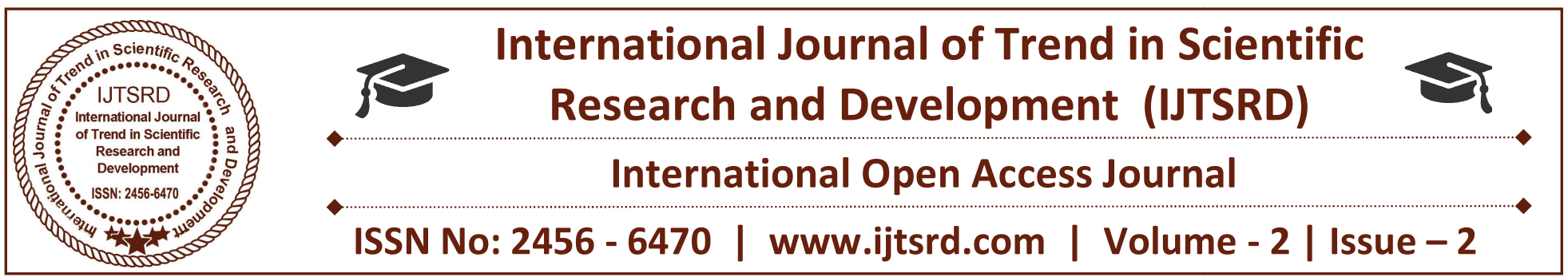

\title{
Orca 4.0.1 Mechanism for Robotics
}

\author{
Rajesh CVS \\ Faculty of Mechanical Engineering Department, \\ Kakinada Institute of Technology and Science, Divili, India
}

\begin{abstract}
This paper describes the Orca 4.0.1 open-source software project which applies Mechanism foundation Software Engineering principles to robotics. The paper gives the industrial aspects of the structure which set it apart from other parallel efforts. Among them are the conversion of a commercial middleware package, minimalist approach to framework design, and a commitment to multi-language multi-platform support. The paper explains our decision to use ice a comparatively modern opening in the field of generalpurpose middleware packages.
\end{abstract}

\section{INTRODUCTION}

This research paper is about Orca 4.0.1 software structure for developing robotic systems. It is one of several systems with similar objectives which are currently under improvement.

The software growth in the environment of robotics currently attract great attention because generating robotic software is difficult and takes lot of time and supposed to be one of the limiting factors in the progress of robotics now a days. It is usually recognized that the key to making progress in this area is software use again, which includes the opensource software and commercially accessible alternatives.

Orca 4.0.1 is one of the open-source frameworks for creating mechanism-based robotic systems. This software shows defining and developing the buildingblocks which can be pieced together to form randomly difficult robotic systems. The different types of systems we are targeting range from particular vehicles to circulated sensor networks. Mainly, we visualize usage patterns typical for both commercial and academic areas.

The research main objective is to support software reuse in robotics. Many factors supply to the success or failure of a reuse initiative. Based on an formal survey of the field, it appears like there is a growing agreement that a successful framework would be:

1) Open-source

2) Modular

3) Circulated under a license in commercial applications;

4) Tested and documented modules

The above requirements are satisfied by Orca 4.0.1 design. Orca 4.0.1 is the open-source under a combination of LGPL and GPL licenses. The framework must containing the following things

5) Flexible

6) Extensible

7) Suitably strong

8) Simple to use

\section{ICE MIDDLEWARE}

To apply a circulated component-based system, one must be able to define interfaces and make a choice of communication machinery. In case of cross-platform procedure involving different operating systems, the software which provides such functionality is typically referred to as middleware. The realities of robotic software development that we consider 
International Journal of Trend in Scientific Research and Development (IJTSRD) ISSN: 2456-6470 support for $\mathrm{C} / \mathrm{C}++$ on Linux to be necessary. This rules out Microsoft's $\mathrm{COM}^{+}$and Sun's activity JavaBeans. Below alternatives are:

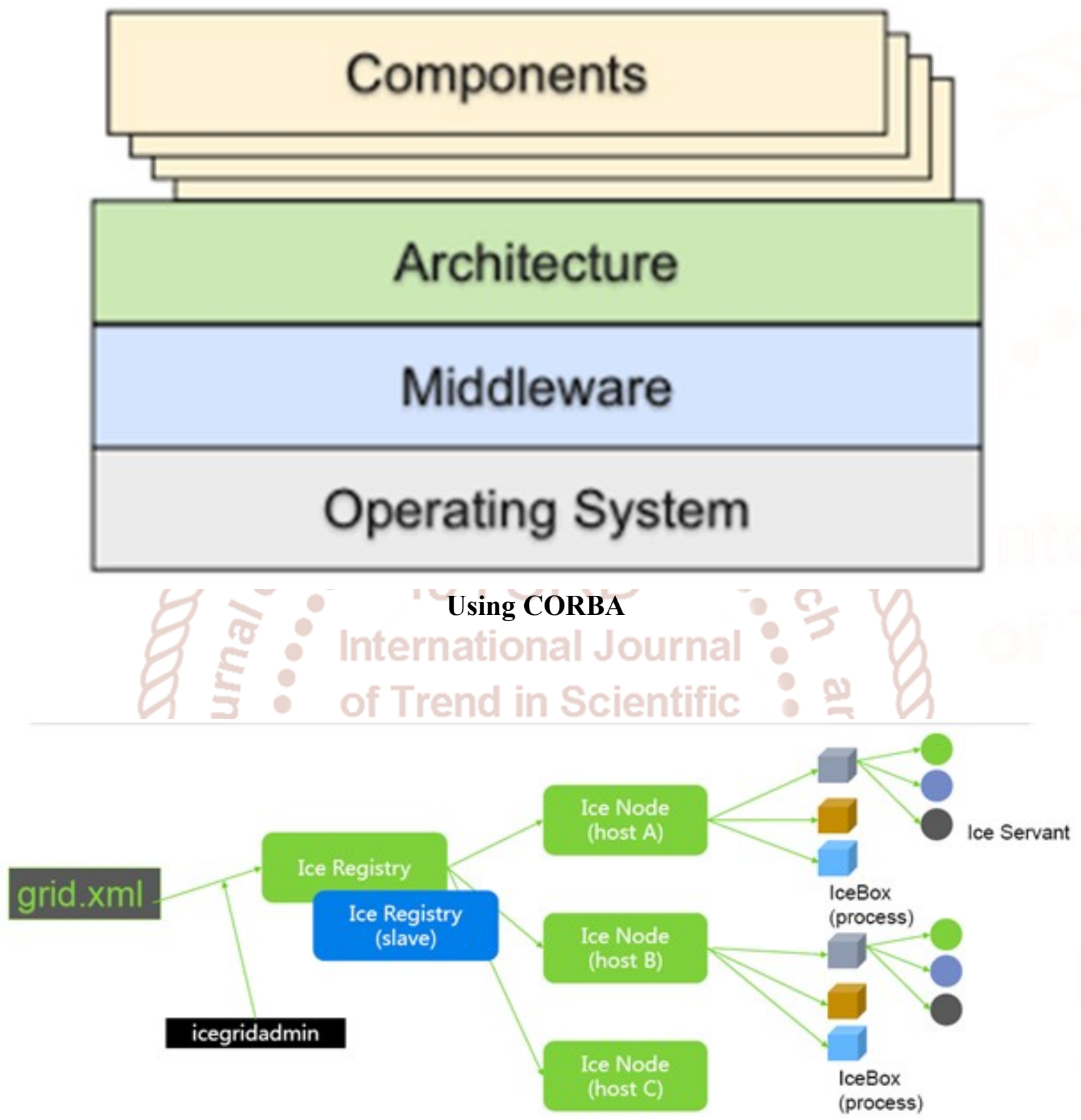

Using Ice 


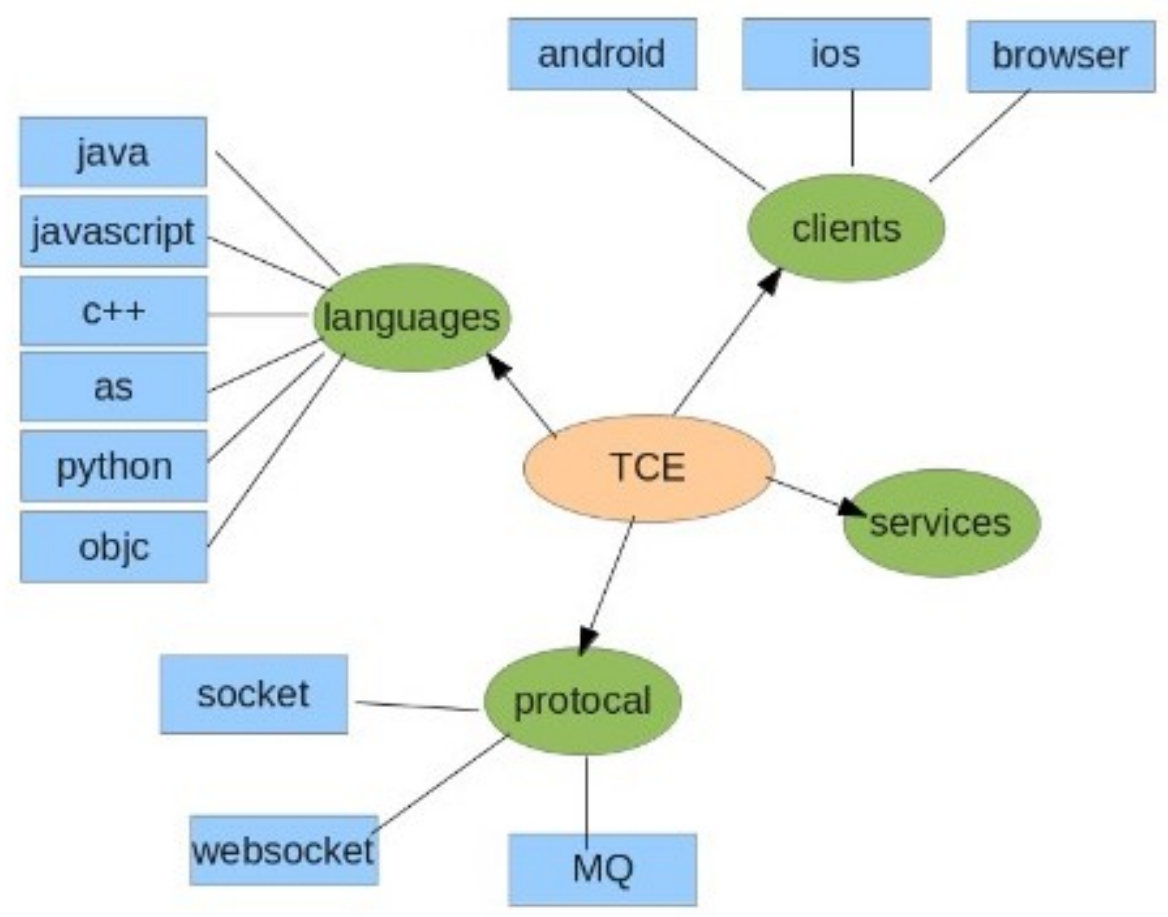

Using XML technologies

Here describes what Ice is and what features it offers.

\section{Ice}

The Internet Communications Engine (Ice) is an implementation of middleware ideas similar in spirit to CORBA.

\section{Ice Services}

Ice middleware comes with a number of services which make available additional functionality. Some of them are used extensively in Orca 4.0.1

\section{CONCLUSION}

We have given that the current state of the Orca 4.0.1 project. Main instructions of current work are knowledge how to use Ice features successfully and improving reliability of obtainable components.

\section{REFERENCES}

1. H. Bruyninckx. Open robot control software: the OROCOS project. In IEEE Int. Conf. on Robotics and Automation (ICRA'01), volume 3, pages 2523-2528, 2001. [5] D.Wheeler. SLOCCount.

2. R.T. Vaughan, B.P. Gerkey, and A. Howard. On device abstractions for portable, reusable robot code. In IEEE/RSJ International Conference on Intelligent Robots and Systems (IROS 2003), pages 2421-2427, 2003.

3. A. Brooks, T. Kaupp, A. Makarenko, A. Orebck, and S. Williams. Towards component-based robotics. In IEEE/RSJ International Conference on Intelligent Robots and Systems (IROS 2005), pages 163-168, 2001. 